\title{
CONF-970591--3
}

\section{NONINVASIVE TESTING OF SOLENOID-OPERATED VALVES USING TRANSIENT CURRENT SIGNATURE ANALYSIS}

\author{
by \\ E. D. Blakeman \\ and \\ R. C. Kryter \\ Oak Ridge National Laboratory \\ Oak Ridge, Tennessee
Manuscript submitted to the
International Conference on Maintenance and Reliability
MARCON 97
May 20-22, 1997
Knoxville, Tennessee

RECEIVED

MAR 181997

OSTI

The submitted manuscript has been authored by a contractor of the U.S. Government under contract AC0596OR22464. Accordingly, the U.S. Government retains a nonexclusive, royalty-free license to publish or reproduce the published form of this contribution, or allow others to do so, for U. S. Government purposes.

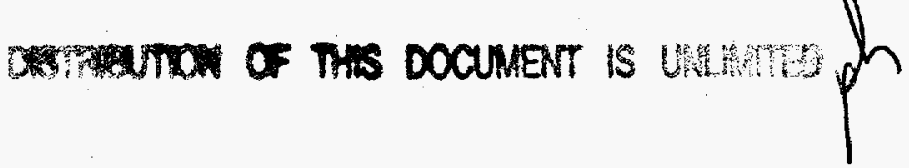

This research was performed at OAK RIDGE NATIONAL LABORATORY, managed by LOCKHEED MARTIN ENERGY RESEARCH CORP. for the U.S. DEPARTMENT OF ENERGY under contract number DE-AC05-96OR22464.

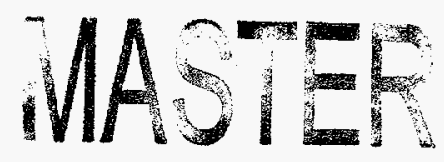




\section{DISCLAIMER}

This report was prepared as an account of work sponsored by an agency of the United States Government. Neither the United States Government nor any agency thereof, nor any of their employees, make any warranty, express or implied, or assumes any legal liability or responsibility for the accuracy, completeness, or usefulness of any information, apparatus, product, or process disclosed, or represents that its use would not infringe privately owned rights. Reference herein to any specific commercial product, process, or service by trade name, trademark, manufacturer, or otherwise does not necessarily constitute or imply its endorsement, recommendation, or favoring by the United States Government or any agency thereof. The views and opinions of authors expressed herein do not necessarily state or reflect those of the United States Government or any agency thereof. 


\section{DISCLAIMER}

Portions of this document may be illegible in electronic image products. Images are produced from the best available original document. 


\title{
NONINVASIVE TESTING OF SOLENOID-OPERATED VALVES USING TRANSIENT CURRENT SIGNATURE ANALYSIS
}

\author{
E. D. Blakeman and R. C. Kryter* \\ Instrumentation \& Controls Division \\ Oak Ridge National Laboratory \\ Oak Ridge, TN 37831-6010
}

\begin{abstract}
A methodology has been developed for in situ diagnostic testing of dc-actuated solenoid-operated valves (SOVs) by analysis of the characteristics of the transient current waveform accompanying valve actuation. This waveform, which is used to identify abnormal operation, is obtained inductively by a small split-core current transformer clamped around one of the valve's solenoid leads. The digitized waveforms are subsequently compared with similar waveforms obtained when the valve was known to be operating normally, and the results used to detect faulty valve operation. This methodology is noninvasive because direct access to the SOV is not required; also, the current transformer has negligible effect on the solenoid current and does not require the lifting of any electrical leads.
\end{abstract}

Analysis is performed by first extracting various waveform "features". A subset of these features is examined by diagnostic algorithms, tuned to identify faults for that valve type, that compare the features with those obtained from a previously acquired waveform for the particular valve under examination. Two methods of analysis have been examined. One utilizes fuzzy logic in an expert system diagnostic. The other performs pattern recognition using an artificial neural network approach. The overall analysis is robust in that it can deal with minor deviations of the waveform shape experienced during repeated operation of a normal valve while successfully identifying abnormal valve operation.

In the present application, analysis has been specialized to SOVs used on space-based systems; however, the methodology has sufficient flexibility to be applicable to a wide variety of valves and other industrial electrically controlled components.

\section{GENERAL DISCUSSION AND EXPERIMENTAL SETUP}

In a previous investigation ${ }^{1}$, monitoring methods with which to detect aging and wear of ac-powered solenoidoperated valves used in safety systems of nuclear power plants utilized the ability to detect armature movement and position from impedance measurements. In the present work, a measurement of the operation of dc-powered solenoid operated valves (SOVs) is obtained by analysis of the characteristics of the transient current waveform accompanying valve actuation. This actuation waveform is extracted inductively using a small, commercially available split-core current transformer clamped around one of the valve's solenoid leads; the signal thus obtained

\footnotetext{
*Retired.
} 
is related to the actual solenoid current by a first-order differential equation. Figure 1 shows the experimental setup used. The analog waveforms are digitized and stored by a personal computer using commercial data acquisition hardware and software.

In the setup shown in Figure 1, a valve is energized by a dc power supply that is rapidly switched on and off at a specified rate by a mercury-wetted relay driven by a pulse generator. This setup permits cyclic operation of the valve at a selected convenient rate (e.g. 1 cycle/s) which affords an easy way to observe the waveform in the lab and to check for repeatability of results. For field application in which the valve would not be operated cyclically, the only instrumentation required would be the clamp-on current transformer, a signal amplifier/filter, and the computer. This instrumentation could be contained in a portable configuration or could be configured in a centralized location where the signals from many valves could be multiplexed into a single analysis system.

Waveforms obtained during valve actuation can be compared with similar waveforms obtained when the valve was known to be operating normally, and the results used to detect faulty valve operation. This methodology is completely noninvasive because the current transformer has negligible effect on the solenoid current and its use does not require the lifting of any electrical leads. Also, the current transformer can be attached to an electrical lead at a location remote from the valve, which is often more accessible than the SOV itself.

\section{VALVE TYPES}

All valves utilized in this investigation were designed to control the flow of nitrogen, helium, or air at pressures up to $6000 \mathrm{psi}$, although typical operating pressures are about $750 \mathrm{psi}$. In this work, results from five valves representing three separate models are presented. They are designated as 79K90221-4, 79K90221-6, $79 \mathrm{~K} 80223-3,79 \mathrm{~K} 80223-9$, and $79 \mathrm{~K} 80225-1$. As indicated by similar prefix numbers (characters preceding the "-"), the first two valves are of the same basic model, the second two of another model, and the fifth of a third model. Valve sizes ranged from $1.68 \mathrm{in}$. diameter (outside case of solenoid) for valves $79 \mathrm{~K} 90221-4,-6$ to 2.23 in. for valves $79 \mathrm{~K} 80223-3,-9$ which weigh approximately four lbs. each. Although the valve models varied in size and internal construction, they all operate in a similar manner. Magnetic forces exerted by energizing the solenoid coil pulls in a plunger acting against the force of a restraining spring. Seals on the plunger poppet seat/unseat to start, stop, or redirect gas flow. Deenergizing the solenoid results in the reverse process.

\section{WAVEFORM SHAPE}

Transient current waveforms acquired in the manner described above have characteristic shapes determined by both a variety of specific valve properties and also by the general operating conditions. In particular, the amplitude vs time waveform generated when the valve is energized has an overall " $M$ " shape that is caused by the inductance change in the coil resulting from plunger motion, coupled with the rapidly changing in-rush current in the coil. Figure 2 shows sample waveforms for two dissimilar valves (79K90221-4 and 79K80223-9) operated under "baseline" conditions (actuating voltage $24 \mathrm{~V}$, ambient temperature $-23^{\circ} \mathrm{C}$, no gas pressure applied to the valve). Although the waveforms are substantially different, they exhibit the same overall characteristic shape. In general, the actuation waveform shape will be altered by valve defects such as impeded or incomplete plunger motion, and reduced plunger return spring force. The waveform is also sensitive to applied voltage and to the valve's operating temperature and gas pressure applied. Similar effects are manifested in the "drop-out" 
waveform obtained when the valve is deenergized. This latter waveform is characterized as a decaying amplitude vs time, but with a discontinuity that occurs when the valve plunger unseats and moves (by virtue of the return spring) to its deactuated position. In our analysis, however, we have found that the waveform obtained upon deenergizing is less sensitive to valve condition and have therefore concentrated on the waveform obtained upon energizing.

\section{FEATURE EXTRACTION}

Analysis of the transient waveforms is performed on various waveform "features"-- such as the amplitudes and times of occurrence of amplitude "peaks" and "valleys", the areas beneath peaks, and appropriate ratios of these quantities. These features are then examined by an expert system/pattern recognition algorithm that has been taught to identify faults for that valve type. The algorithm compares the features with those obtained from a previously acquired baseline waveform for the valve under examination and determines if the valve is actuating properly. The feature extraction/quantification methodology must be robust so that it can deal with minor deviations of the waveform shape experienced during repeated operation of a normal valve, while still successfully identifying truly abnormal operation.

Referring again to Figure 2, both of the waveforms are observed to have two peaks and a narrow valley between them. The first peak corresponds to the begiming of rapid plunger movement during the initial phase of the current inrush transient. The valley occurs when the plunger has completed its stroke, and the second peak represents a maximum in the $\mathrm{AC}$ portion of the signal as the current increases to its steady-state value during the latter part of the transient. This is the general form for all the valves we have examined; we currently extract eleven features, of which six are independent of amplifier gain, as follows:

1) time to reach Peak 1

2) time to reach Valley $[\mathrm{tV}]$

3) time to reach Peak 2

4) (Peak 1 amplitude)/(Peak 2 amplitude) [rPP]

5) (Peak 1 amplitude)/(Peak 1 amplitude - Valley amplitude) [rPV]

6) (Area beneath Peak 1)/(Area beneath Peak 2)

For initial demonstration purposes, diagnostics have been performed using only three of these features: the time to reach the "valley" (2), the ratio of the two peak amplitudes (4), and the ratio of the amplitude of the first peak to the difference between the peak and valley amplitudes (5). The shorthand notation for these features is $t V$, $\mathrm{rPP}$, and rPV, respectively. It is not difficult to see that an off-normal situation will change the values of these features. For example, a broken, weak, or "loose" (compression adjusted to a lower value) return spring will result in a smaller tV since the valve will close more quickly. Also, a smaller rPP, and a slightly smaller rPV than for the normal baseline case will typically result from this situation. As another example, if the valve plunger does not fully stroke, the amplitude of the valley will be high, thus increasing the value of rPV; however, tV will change very little, since the valve still actuates in about the same time as the normal case. Figure 3 shows an example of waveforms obtained from a valve with these defects induced as compared to those from a valve displaying normal baseline operation. Different diagnostics methodologies to distinguish between the various cases are available. Two methodologies that have been examined and are discussed in a later section are fuzzy logic and artificial neural networks.

In addition to the above features, we are also considering other waveform comparison possibilities, among which 
are waveform correlation, Fourier decomposition, and moments calculations ${ }^{2}$. These calculations, all of which are used to determine an indication of waveform shape, could be used for initial waveform screening, or included with the features described above. Of the above methodologies, the moments calculation presently shows the most promise. In this approach, the waveform is considered to be a function $f(t)$ of time $t$, normalized to unit area. The moments are given by

$$
\mu_{n}(t)=\sum_{i=1}^{K}\left(t_{i}-m\right)^{n} f\left(t_{i}\right)
$$

where $K$ is the number of points and $m$ the mean value (calculated by setting $m=0, n=1$ in the above equation). The second and third moments, $\mu_{2}(t)$ and $\mu_{3}(t)$, were observed to be especially sensitive to the simulated valve defects, suggesting that features based on these calculations could provide additional discrimination capability.

\section{FEATURE NORMALIZATION}

A difficulty encountered in the application of any diagnostic analysis to the waveform features is the variation in the waveform characteristics obtained for individual valves of the same type. Like a human being, each valve has unique physical characteristics and will thus exhibit a unique actuation waveform even under the exact same operating conditions as another valve bearing the same type number. Figure 4, for example, shows two distinctly different actuation waveforms for valves of similar type (among valves of different types, the waveforms are considerably more disparate, as is indicated by Figure 2). It is clear that valve 79K90221-4 actuates more quickly than valve $79 \mathrm{~K} 90221-6$, thus indicating that it has a weaker return spring or exerts a stronger magnetic force when energized, which can result from a larger inductance (the inductance of the solenoid in valve $79 \mathrm{~K} 90221-4$ was measured to be about $30 \%$ higher than valve $79 \mathrm{~K} 90221-6$ ). Similar differences, but to a lesser degree, are shown in Figure 5 for actuation waveforms for valves 79K80223-3 and 79K80223-9.

In our diagnostics application it is assumed that one valve of a particular type has been utilized to generate a database of simulated defects for testing valves of its same type. For the valves to be tested it is assumed that the only data available are for normal baseline operation. Since, as shown above, each valve has a unique set of operating characteristics, data from one valve must be used to assess problems in another valve that will almost certainly have different operating characteristics. The approach we used to circumvent this problem is to always normalize the waveform features to those obtained for that valve under normal baseline operation. Thus, we look at relative changes in the waveform features, rather than at the absolute values of the features themselves. This approach, to be successful, requires that the waveform characteristics of valves of similar types change in the same way as a result of a particular defect. As an illustration, Figures 6 and 7 show plots of the normalized values of $\mathrm{tV}, \mathrm{rPP}$, and $\mathrm{rPV}$ for valve $79 \mathrm{~K} 80223-3$ for 60 valve actuations that included simulation of valve defects and baseline cases ( 24 actuations). The defects included:

1. Decreased operating voltage: 0.5 and 1.0 volt ( 4 actuations each)

2. Increased operating voltage by 0.5 and 1.0 volt (4 actuations each)

3. Return spring loosened $1 / 12$ and $1 / 6$ turns (4 actuations each)

4. Plunger motion obstructed by 1.5 and 3 mil shims ( 4 actuations each)

5. Plunger motion impeded ( $\sim 1$ mil strip applied to plunger stem) (4 actuations) 
The decreased/increased voltage to the valve coil can be considered as a defect, such as a shorted turn, in the coil or as an off-normal operating condition. The reason for the larger number of baseline cases is that the tests were performed in groups of 4 following the defect simulations to ensure consistency of performance throughout the testing sequence.

The data are plotted in a two-dimensional form with one feature on each axis. In each case, the feature value was normalized to the value obtained from the first (baseline) actuation. Here it can be seen that, for a single valve, the various valve defects separate the data into distinct groupings or clusters. A nearly identical plot can be made for a similar valve, $79 \mathrm{~K} 80223-9$, provided that the feature values are again normalized to the first baseline actuation for that valve. Two conclusions can be drawn from these results. First, the features vary in a consistent and distinctly different manner for each of the defects simulated. Second, the relative change for each of the defects is about the same for the two valves shown. Similar results were observed for the other valves studied.

\section{EFFECTS OF OPERATING CONDITIONS}

For operating conditions other than the baseline conditions, the effects on the actuation waveforms must be well understood, since conditions can be expected to vary considerably in actual practice and are not ordinarily controllable. Ambient temperature effects were investigated by acquiring actuation data with valves located in a containment having a controlled temperature. In general, an increase in temperature will increase the dc resistance of a valve solenoid and thus decrease its current draw. Figure 8 shows two actuations of valve $79 \mathrm{~K} 90221-4$ at ambient temperature and $30^{\circ} \mathrm{C}$ above ambient, with all other operating conditions held the same. As expected, the waveform at the increased temperature is reduced in amplitude. Also, relevant to ambient temperature, the valve at the elevated temperature actuates slightly later and the ratio of the two peaks (rPP) is increased slightly. However, if the waveforms obtained at this temperature are normalized to the first case for which there are no simulated defects, similar separation of defects are observed. From this and additional results using other valves, it can be concluded that the actuation waveforms vary in the same manner with valve defects at any reasonable temperature, which should be measurable to within $\sim 3^{\circ} \mathrm{C}$ by placing a small dc voltage source across the valve solenoid and measuring the current prior to actuation.

It was shown previously that the actuation waveform shape varies significantly with the valve temperature. Also it is known that temperature extremes are encountered in the normal operational environment of the valves. Thus, a measurement of temperature to an accuracy of a few degrees centigrade should accompany the waveform measurements. Fortunately, this measurement may be conducted without the addition of instrumentation to the valve. The electrical resistance of the copper wire used to construct the solenoid is known to increase linearly with its temperature. For small temperature differences it follows that

$$
I=I_{0}\left[1-\alpha_{0}\left(T-T_{0}\right)\right]
$$

where $T_{0}$ is a reference temperature and $\alpha_{0}$ is the thermal coefficient of conductivity for copper at the temperature $\mathrm{T}_{0}$ (for $\left.20^{\circ} \mathrm{C}, \alpha_{0}=0.0039 \mathrm{o}^{\circ} \mathrm{C}^{-1}\right)^{3}$. Figure 9 shows the current for each of two valves as a function of temperature for a source voltage of $1 \mathrm{~V}$. The relationship is seen to be approximately linear as predicted by the above equation. Also, if the above value for $\alpha_{0}$ is used, the predicted currents at $60^{\circ} \mathrm{C}$ for valves $79 \mathrm{~K} 80225-1$ and $79 \mathrm{~K} 80223-3$ are $47.0 \mathrm{~mA}$ and $34.4 \mathrm{~mA}$, respectively, within a few percent of the measured values. 
The data described thus far were acquired without pressurizing the valves. Actual testing of the valves may be performed with or without pressurization. It is important therefore, to understand how the valves behave under pressure and whether valve defects will produce the same deviations with/without pressurization. Testing performed at relatively low pressures (e.g. $500-750 \mathrm{psi}$ ) determined that the effect on the operational waveform is both small and predictable. For example, a pressure of 500 psi on valve $79 \mathrm{~K} 80225-1$ delayed the valve closing by about $2 \mathrm{~ms}$ and caused barely perceptible differences in the height of the waveform peaks. This suggests that for typical operating pressures, if the pressure is known to some reasonable accuracy, e.g. 100 psi, the effects can be readily taken into account.

\section{DIAGNOSTIC ANALYSIS USING WAVEFORM FEATURES}

For this work we examined two different but complementary types of automated analysis that utilize as input the features extracted from the actuation waveforms as discussed in the previous section. The first is an expert systems approach incorporating fuzzy logic. This approach utilizes a set of heuristic "if - then" rules to determine valve condition. Fuzzy logic allows for uncertainty in the input values by permitting those values to be expressed with linguistic quantifiers such as "large", "small", "few", "many", etc.

The second methodology is a trainable pattern recognition algorithm that maps input patterns (combinations of feature values) into different output configuration determinations (e.g. normal actuation, loose spring, etc.). The algorithm, referred to as a neural network, is trained using data acquired under known normal and abnormal valve conditions so that when a set of features from an unknown valve condition is input, it will respond with the correct assessment. For both methodologies commercially available software packages were used ${ }^{4,5}$.

For the fuzzy logic approach, fuzzy rulebases were developed to determine valve defects including a) failure of the valve to actuate, b) weak or broken valve spring, c) impeded valve motion, d) high or low solenoid voltage. Each rulebase is slightly different from the others since each valve type responds somewhat differently to the various simulated defects. In general, about 20 to 25 rules applied to the three features $\mathrm{tV}, \mathrm{rPP}$, and $\mathrm{rPV}$ are sufficient to distinguish the valve defects. Each feature value is described by one of five fuzzy descriptor values, VERY LOW (VL), LOW (L), MEDIUM (M), HIGH (H), or VERY HIGH (VH). The mapping of the descriptors to actual numbers is controlled by the user through the input of range values. Outputs are divided into three descriptors, LOW (L), MEDIUM (M), or HIGH (H). Thresholds are set in the upper part of the MEDIUM range such that if the output for a particular defect is above that value, then the valve is judged to exhibit abnormal operation.

For the neural network approach, a neural network model was implemented for each of the different valve types. Each model used three inputs which corresponded to the features $\mathrm{tV}, \mathrm{rPP}$, and $\mathrm{rPV}$ and six binary outputs which corresponded to the base case and defective valve states (base case, incomplete valve closure, low solenoid voltage, high solenoid voltage, impeded valve motion, loose/weak return spring). Each input vector therefore consisted of three values and each output vector consisted of six values, one of which was 1 , indicating the condition state of the valve, and the others 0 .

Training data for valve types $79 \mathrm{~K} 80223$ and $79 \mathrm{~K} 80221$ were comprised of the information derived from 52 of the 60 waveforms discussed earlier (cases for the 0.5 volt low and 0.5 volt high power supply voltages were 
omitted). Best results were obtained when five hidden nodes were used. Thus, each network consisted of three input nodes, five hidden nodes, and six output nodes. Training was accomplished by presenting the input/output vectors to the network in random order and adjusting the connection weights in accordance with the backpropagation algorithm until agreement between the desired and actual outputs was achieved. Results were tested by imputing the training to the network. The approach used was to select the highest output vector component and see if it was approximately 1.0 in the correct location. A "suspicious" reply was assumed if this value was 0.6 or less.

\section{RESULTS OF DIAGNOSTIC ANALYSIS}

The neural network approach was very successful. All 52 of the training vector pairs for valves $79 \mathrm{~K} 80223-3$ and $79 \mathrm{~K} 90221-4$ resulted in correct identification. This is not surprising, since it is expected that data used for training would result in a correct response. However, data from valve $79 \mathrm{~K} 80223-9$ also resulted in correct identification in all cases but one. For valve 79K90221-6, 48 of the 52 cases were correctly identified (two were suspicious). The four cases misidentified were those with the operating voltage reduced by 1 volt; these were interpreted as an "impeded plunger". Examination of the three features used shows that this confusion is understandable since they are similar in value for the two situations. Results from 52 test cases for valve $79 \mathrm{~K} 90221-4$ heated to $30^{\circ} \mathrm{C}$ above ambient were also tested. Remarkably, all 52 of the cases were correctly identified (two were "suspicious" however). Overall, it is concluded that these results indicate that the method is quite successful, in that it was able to generalize results from one valve to a different valve of similar type and to different operating conditions!

Somewhat less successful results were obtained for the fuzzy logic rule-based approach. For valve 79K80223-3, six of the 52 cases were misidentified. These include four cases in which high voltage $(+1 \mathrm{~V})$ was misinterpreted as low return spring pressure. Examination of the data showed that the features behave in a similar way for the two cases; thus, this outcome is predictable. Similar results were obtained for valve $79 \mathrm{~K} 80223-9$ using the same rulebase. Of the 52 cases, seven were misidentified, again including the four cases in which the high voltage is misinterpreted as low return spring pressure. Better results were obtained with valves $79 \mathrm{~K} 90221-4$ and -6 . For valve $79 \mathrm{~K} 90221-4$, of the 52 cases examined, two were misidentified and one was ambiguous. For valve $79 \mathrm{~K} 90221-6$, which was examined using the same rulebase, 48 of the 52 cases were correctly identified. The four misidentified were the four cases with low voltage (misinterpreted as an impeded plunger). These latter results are consistent with the neural net response and are readily explainable, since the features behave in almost exactly the same manner for the two situations.

\section{SUMMARY \& CONCLUSIONS}

This work has demonstrated that by analysis of electrical waveforms obtained during normal actuation of an SOV, the operational health of the valve can be assessed in a completely noninvasive manner. Operational flaws in valve operation were simulated and shown to be readily detectable using either of two diagnostic methodologies. Results obtained from one valve can be used to detect operational flaws in another valve of the same type for differing operating conditions (e.g., higher temperature). It was shown that valve current signatures are highly dependent on temperature, but that the temperature can be obtained readily from a resistance measurement of the valve without the addition of instrumentation. It is anticipated that two measurement strategies might be employed. First, the system could provide immediate indication of a valve that has either failed or is operating in a degraded mode near failure. Also, the system could be used to acquire a long-term 
database covering many valve actuations. This database could be examined in a statistical sense for gradual or sudden degradations of response that might indicate a proximity to failure.

A system utilizing the above methodology would use a minimum of equipment. It could either be portable or integrated into an existing data acquisition system and automated. In either case, such a system would afford remote diagnosis of inaccessible components under operating conditions. This permits early detection of faulty components as well as considerable savings in the time and effort required to remove components for bench-top testing. For these reasons, the methodology is expected to be useful in the areas of predictive maintenance and reliability assessment. Although, in the present application, analysis has been specialized to SOVs used on spacebased systems, the methodology has sufficient flexibility to be applicable to a variety of valves and other industrial electrically controlled components as a part of an overall system for diagnostic monitoring during realtime operation. It is expected that such a diagnostic system, based on fuzzy logic and/or the neural network approaches, would utilize a large database and sufficient calculated features to prevent ambiguous responses and thus provide for fault-tolerant assessments.

\section{ACKNOWLEDGEMENTS}

Funding for this work was provided by the National Aeronautics and Space Administration, Kennedy Spaceflight Center (NASA-KSC).

\section{REFERENCES}

1.R. C. Kryter, Aging and Service Wear of Solenoid-Operated Valves Used in Safety Systems of Nuclear Power Plants, Evaluation of Monitoring Methods, NUREG/CR-4819 (ORNL/TM-12038), Vol. 2 (1992).

2. R. C. Gonzalez, P. Wintz, Digital Image Processing, Addison-Wesley ( 1987).

3. D. G. Fink, H. W. Beaty, Standard Handbook for Electrical Engineers, Eleventh Edition, McGraw-Hill, pp. $4-5,(1978)$.

4. FC110 Development System User's Manual, Togai InfraLogic, Inc., 30 Corporate Park, Suite 107, Irvine, CA 92714 (1990).

5. NeuralWorks Professional II/PLUS and NeuralWorks Explorer, NeuralWare, Inc., Building IV, Suite 227, Penn Center West, Pittsburgh, PA 15276 (1991). 
Solenoid Operated Valve [SOV]

To DC Power Supply

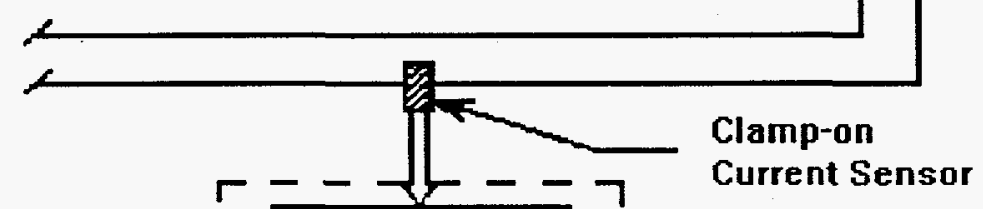

Display \& Keyboard

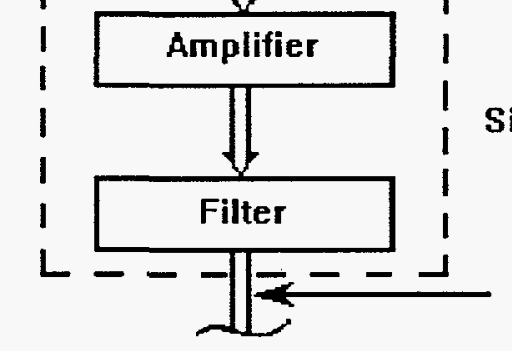

Filtered Analog Sensor Data

See Note Below

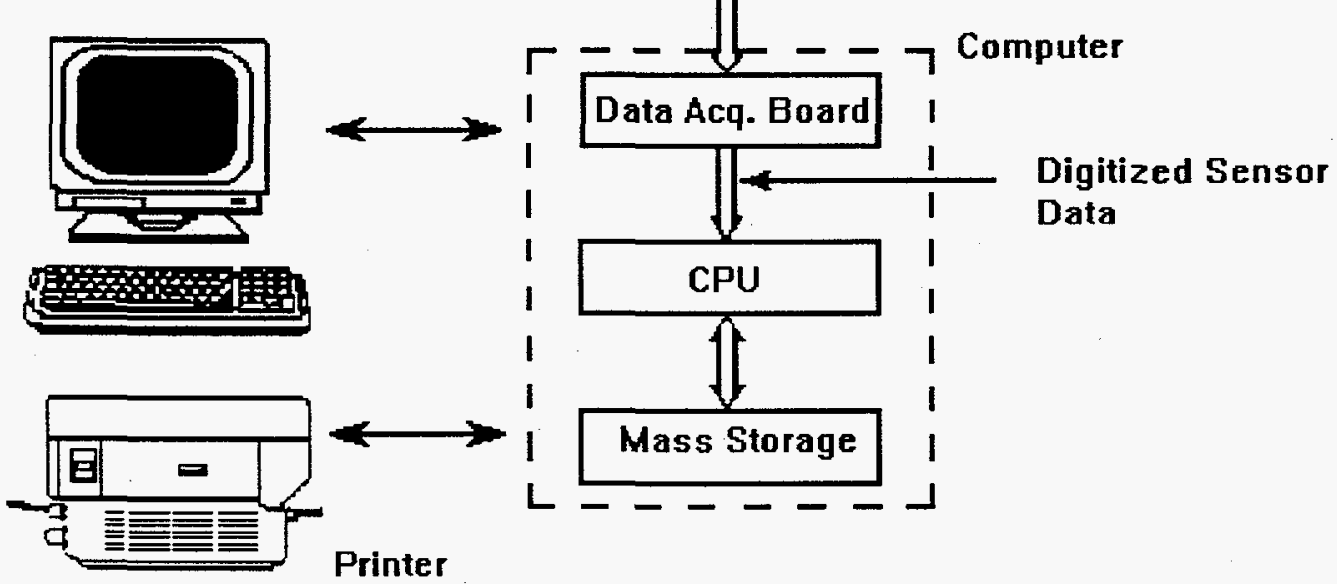

Note: The computer can be placed a considerable distance from the SOV and the signal conditioning system. Likewise, the clamp-on current sensor need not be located close to the SOV under test.

Figure 1 Experimental Setup for SOV Testing 


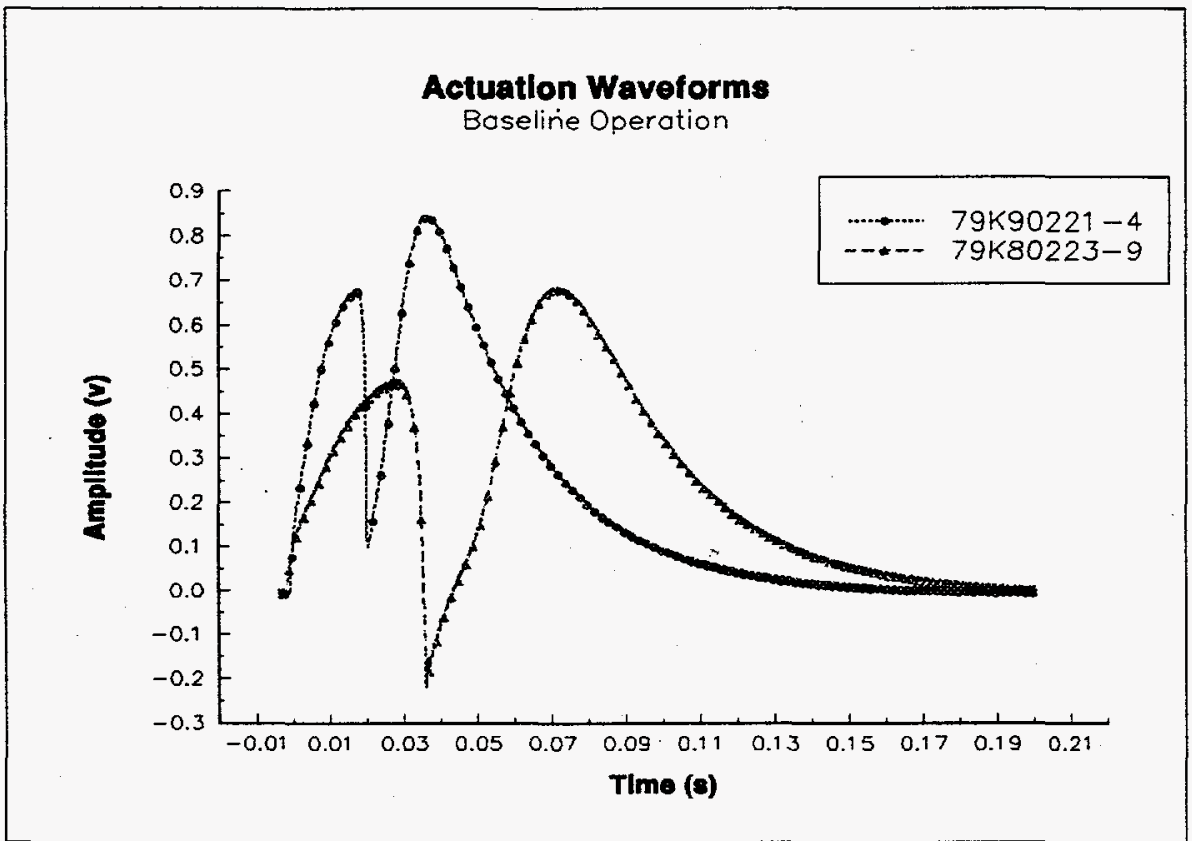

Figure 2 Actuation waveforms for two different valve types.

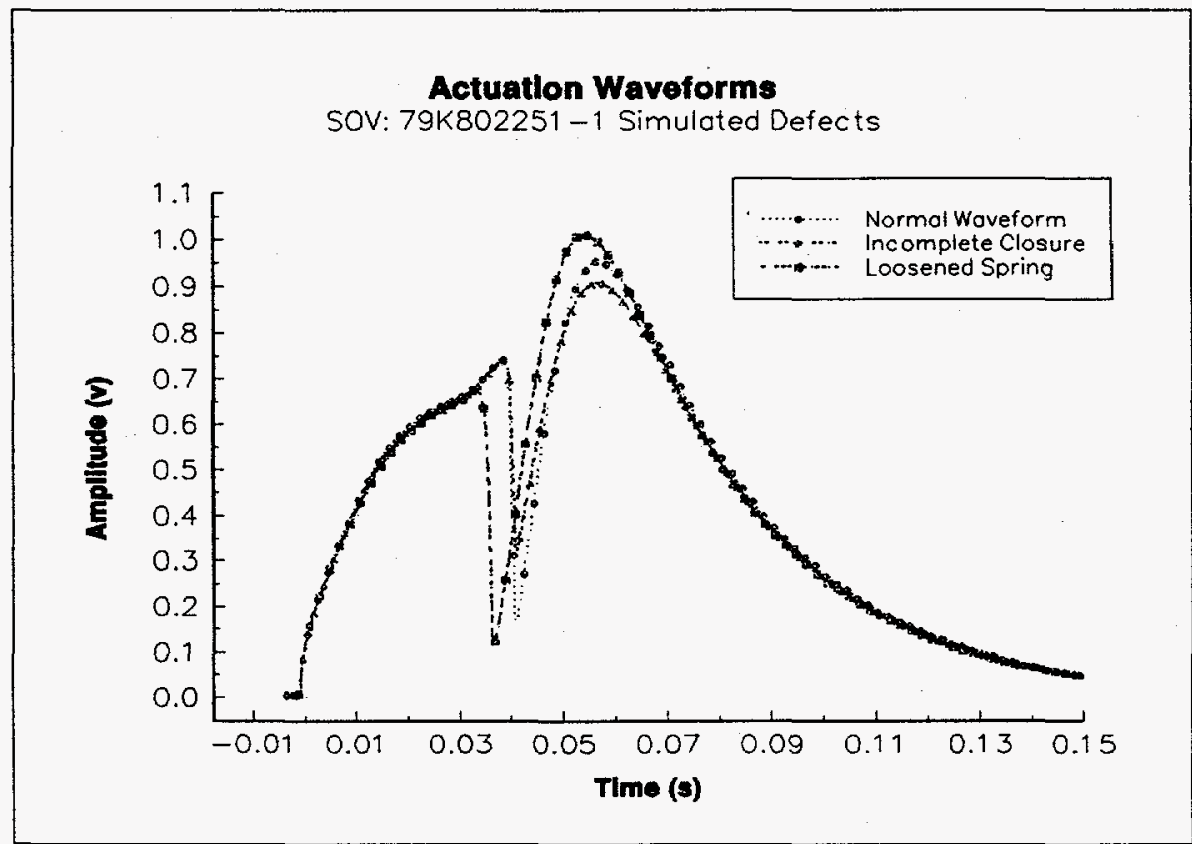

Figure 3 Actuation waveforms for simulated defects in valve 79K80225-1. 


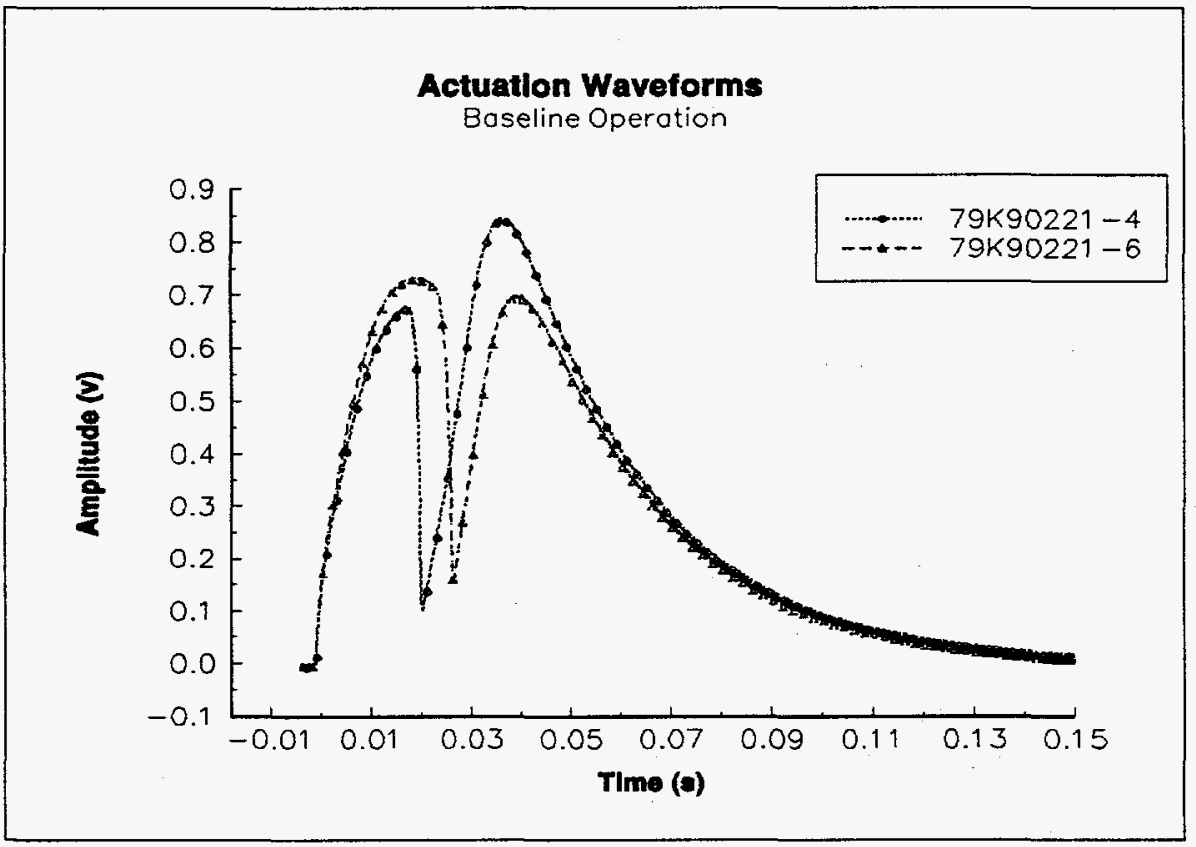

Figure 4 Actuation waveforms illustrating individual differences for baseline operation of valves of the same type.

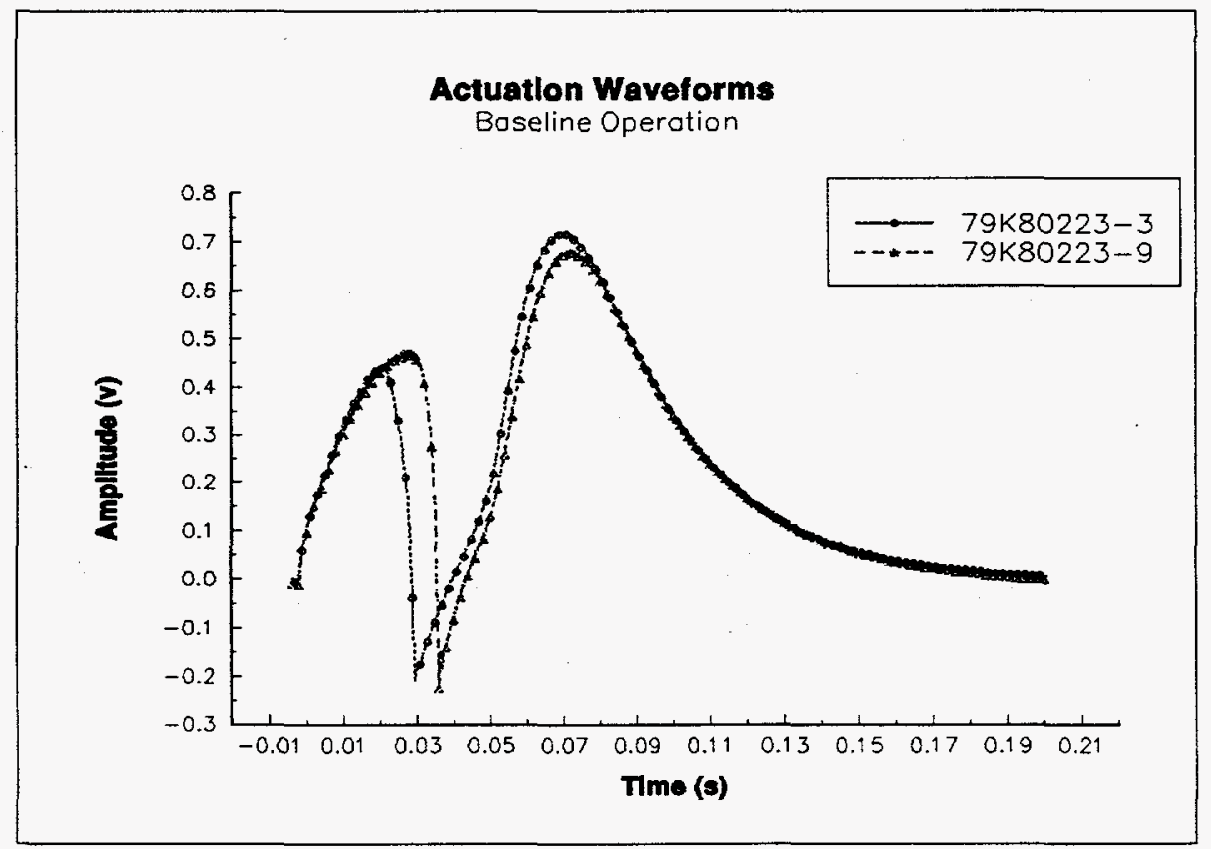

Figure 5 Actuation waveforms for baseline operation of two other valves of the same type. 
Normallzed Features (rPV vs rPP)

SOV: 79K80223-3 Simulated Defects

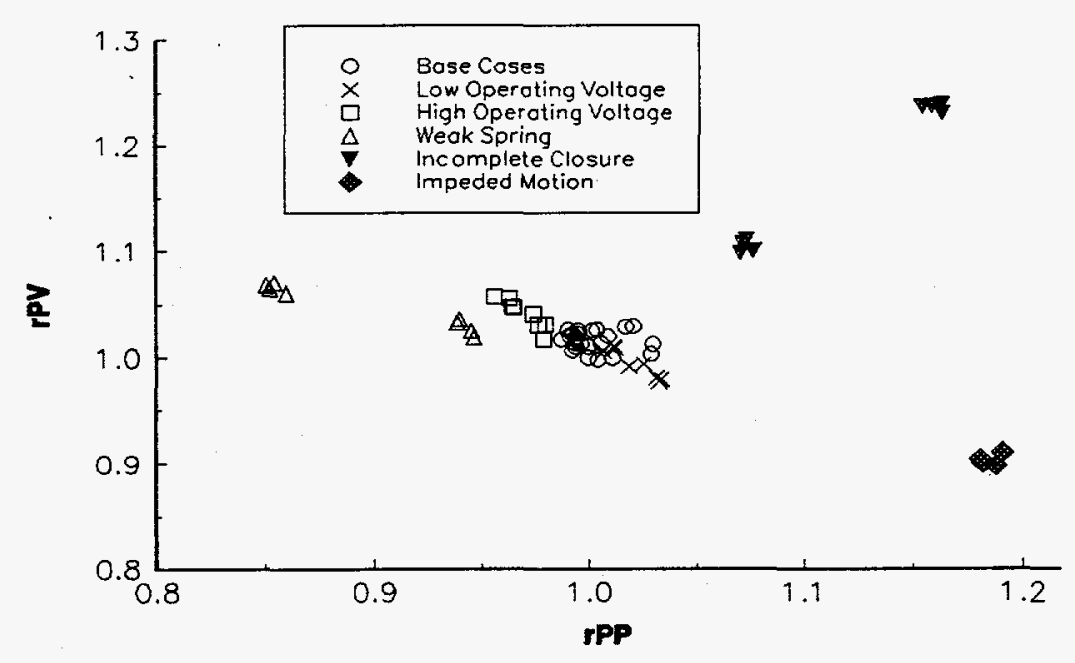

Figure 6 Normalized features (rPV vs rPP) for valve 79K80223-3.

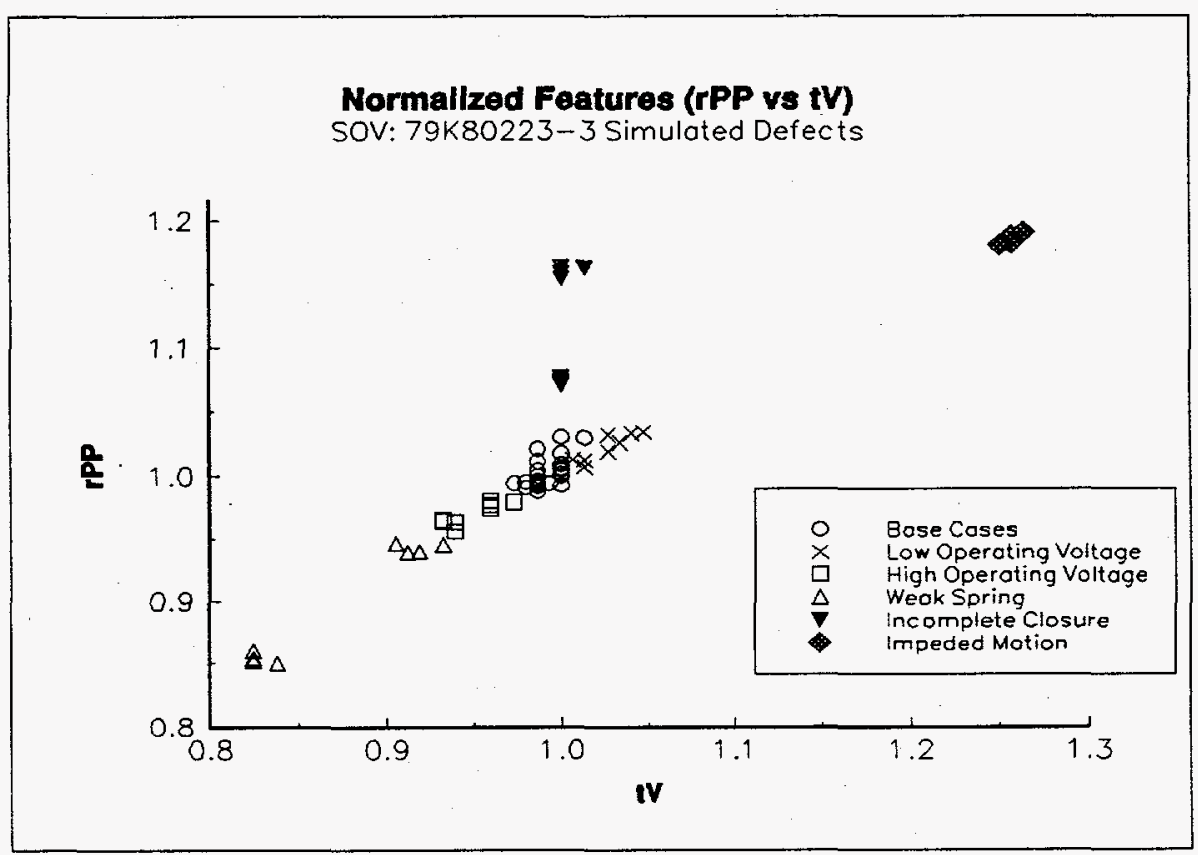

Figure 7 Normalized features (rPP vs tV) for valve 79K80223-3. 


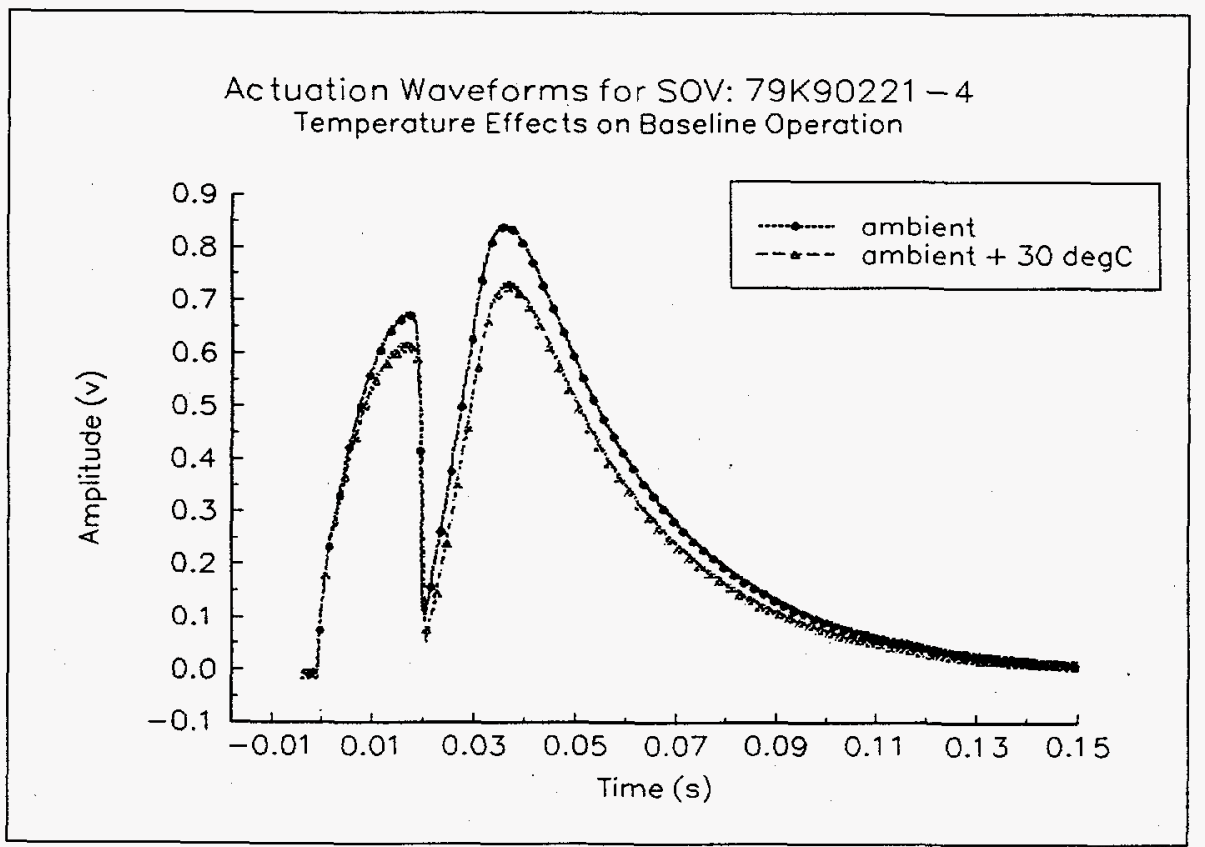

Figure 8 Temperature effects on actuation waveform for baseline operation of valve 79K90221-1.

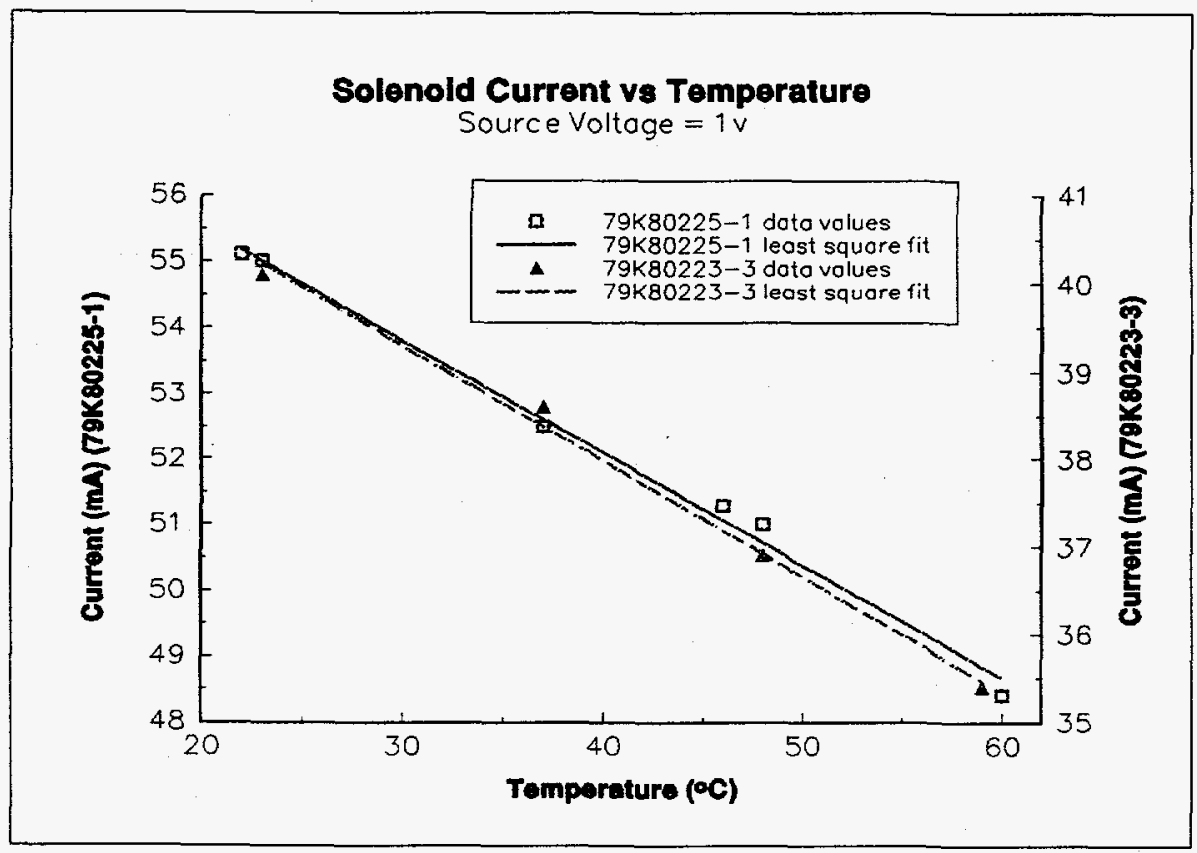

Figure 9 Temperature effects on steady-state solenoid current in two valves. 